\title{
Chronic Dietary Administration of 2-Deoxy-D-Glucose Does Not Compromise Neurobehavioral Functions at Tumor Preventive Doses in Mice
}

\author{
Saurabh Singh', Mayank Kumar1, Kailash Manda1*, Seenu Haridas' ${ }^{1}$, Anant Narayan Bhatt ${ }^{1}$, \\ Daman Saluja $^{2}$, Bilikere S. Dwarakanath ${ }^{*}$ \\ ${ }^{1}$ Division of Radiation Biosciences, Institute of Nuclear Medicine and Allied Sciences, Delhi, India \\ ${ }^{2}$ Medical Biotechnology Laboratory, Dr. B.R. Ambedkar Center for Biomedical Research, University of Delhi, \\ Delhi, India \\ Email: ${ }^{* k a i l a s h m a n d a @ g m a i l . c o m, ~}{ }^{*}$ dwarakanathdrbs@gmail.com
}

Received 30 June 2015; accepted 21 August 2015; published 24 August 2015

Copyright (C) 2015 by authors and Scientific Research Publishing Inc.

This work is licensed under the Creative Commons Attribution International License (CC BY).

http://creativecommons.org/licenses/by/4.0/

(c) (i) Open Access

\begin{abstract}
Beneficial effects of dietary energy restriction (DER), including extension of life-span, reduction in cancer risk, anti-cancer effects and decrease in age related neurodegenerative diseases have been well established. Given that DER is difficult to implement in humans due to practical constraints, development of energy restriction mimetics (ERMs) is considered as a suitable alternative. Our recent studies have established the anti-tumor effects of the dietary administration of the glycolytic inhibitor 2-deoxy-D-glucose, a potential ERM, an alternative to DER; without any adverse effects on general physiology. Since functioning of the brain is critically dependent on glucose, we investigated the effects of chronic dietary 2-DG administration on the behavioural outcome in mice. Our findings based on a battery of neuro-behavioural tests clearly suggest that the chronic dietary administration of 2-DG that appreciably impairs the process of tumorigenesis has no adverse effect on the cognitive, affective and sensory-motor functions. Together with the maintenance of normal physiology reported by us earlier, these observations strengthen the potential of dietary 2-DG as a safe cancer preventive strategy.
\end{abstract}

\section{Keywords}

Glycolysis, Energy Restriction Mimetics (ERMs), 2-Deoxy-D-Glucose (2-DG), Tumor Prevention,

${ }^{*}$ Corresponding authors. 


\section{Neuro-Behaviour}

\section{Introduction}

Dietary energy restriction (DER) has been well established to prolong life with reduced age related diseases including cancer. Since it is practically difficult to implement such a dietary regimen in humans, energy restriction mimetics (ERMs) have been considered as a suitable alternative [1] [2]. In the recent past, numerous studies have focused on the development of these ERMs, which cause effects similar to DER without reducing the actual dietary/calorie intake. Several metabolic modifiers such as resveratrol, metformin and 2-deoxy-D-glucose (2-DG) have shown potential to produce tumor inhibitory and anti-aging effects [3]-[7]. Since tumors show high dependency on glycolysis as a result of metabolic reprogramming (Warburg effect) associated with tumorigenesis [8] [9], there is a considerable amount of interest in exploring 2-DG as a potent anti-neoplastic agent [7] [10]. 2-DG is a remarkable ERM as it simulates DER phenotype which includes gluco-regulatory and other energy modulation effects at systemic as well as cellular levels [6] [7] [11] [12]. At the systemic level, 2-DG influences metabolic regulation by reducing the systemic glucose availability, thereby causing a metabolic stress leading to the activation of multiple stress adaptation mechanisms in normal tissue/cells, while enhancing the death of tumor cells by inhibiting the up-regulated glucose metabolism [6] [7] [13] [14].

2-DG is an analogue of glucose which is phosphorylated to 2-deoxy-glucose 6-phosphate by hexokinase similar to glucose but not metabolized further thereby inhibiting the glycolysis and causing depletion of energy (ATP levels) [10]. 2-DG has been shown to differentially enhance the damage caused by radiation and chemotherapeutic drugs in cancer cells with high rates of glycolysis and therefore has been suggested as an adjuvant to radiation and chemotherapy of tumors [14] [15]. Several preclinical and certain clinical trial studies suggest that 2-DG can be used alone or in combination with chemo- and radiotherapy for cancer treatments [16]-[20]. Clinical studies in patients with cerebral gliomas have shown that the combined treatment of orally administered 2-DG with hypo-fractionated tumor irradiation provides modest increase in survival with improved quality of life and is associated with minimal acute or late toxicity [21] [22]. Although earlier studies using therapeutic doses of 2-DG to achieve local tumor control have not been very successful due to undesirable toxicity [23] [24], we have recently shown that chronic dietary administration of $2-\mathrm{DG}$ at low doses $(0.2 \%$ and $0.4 \% \mathrm{w} / \mathrm{v}$ in drinking water) significantly reduces the growth of implanted tumors and chemical induced tumorigenesis in mice suggestive of cancer preventive effects [25] [26]. Encouragingly enough, this dietary 2-DG administration did not result in significant changes in the food intake, body weight etc., but elicited positive physiological changes similar to DER such as mild reduction in body temperature, blood glucose and serum insulin levels [25]. Reduction in the systemic oxidative stress, induced angiogenesis and inhibition of glucose metabolism appear to be contributing factors responsible for the inhibition of tumorigenesis [25] [26].

Since quality of life is an important consideration in cancer therapy and prevention, it is important to evaluate the effects of ERMs on general physiology as well as behaviour, for safety and successful implementation. Moreover, since the brain mainly depends on glucose metabolism for its function, it is desirable that chronic dietary administration of glycolytic inhibitor like 2-DG be evaluated for possible functional disturbances. The oral safety of 2-DG with respect to neurobehavioral functions has not been systematically investigated in animal models. To the best of our knowledge there has been only one behavioral toxicity study that indicated the anticonvulsant and antiepileptic effects of 2-DG in rats without alterations in spatial learning and memory or transient reversible decrease in exploratory activity [27] [28]. The present study was undertaken to investigate the effects of chronic dietary administration of 2-DG on cognitive, affective and motor functions in mice. Results show that dietary administration of 2-DG, which results in the inhibition of tumorigenesis does not compromise the behaviour of mice.

\section{Material and Methods}

\subsection{Animals and 2-DG Administration}

Eight to ten week old female Swiss albino strain "A" mice weighing 22 - 28 g were obtained from the in-house experimental animal facility (EAF). All mice were maintained at $23^{\circ} \mathrm{C}-25^{\circ} \mathrm{C}$ under a constant 12 -hour light/ 
12-hour dark cycle. These mice were randomly divided into three groups ( $\mathrm{N}=6$ /group); Control, $0.2 \%$ 2-DG and $0.4 \%$ 2-DG (w/v). Control mice were given normal drinking water, whilst the treatment groups were given $0.2 \%$ and $0.4 \%$ 2-DG w/v in their daily drinking water. All mice were given standard rodent feed ad libitum (Golden Feeds, Delhi). The 2-DG was chronically administered for 3 months (90 days) to both the groups before any behavioural testing and was also continued during the test period. Body weight of mice was monitored throughout the span of the study. All behavioural tests were performed during the light phase between 10 a.m. and 4 p.m. The protocols used were approved by the Committee on the Ethics of Animal Experiments of the Institute of Nuclear Medicine and Allied Sciences (INMAS), Defence Research and Development Organization (DRDO) (Institutional Ethical committee number under which this study has been approved is INM/IAEC/2011/ 08/001).

\subsection{Open Field Test (OFT)}

For evaluating general locomotor activity, stress and anxiety-like behaviour of mice, open field test (OFT) was performed in an inescapable square plexiglass box of $40 \times 40 \mathrm{~cm}^{2}$ [29]. It was divided into 16 equal squares or grids. The central 4 grids were considered to be the center, and the rest were assigned as the periphery. The test was performed as previously described [30]. Briefly, the mice exploratory activity was observed and recorded for 5 minutes in the OFT arena. The behaviour was video-monitored and analyzed automatically by using the ANYmaze ${ }^{\mathrm{TM}}$ Software (Stoelting Co., USA). The maze was cleaned up carefully after every individual mouse tested. During the observed time period, number of squares crossed, vertical movements, and latency to enter the center, time spent in the center, number of grooming episodes and number of fecal boli passed were recorded.

\subsection{Elevated plus Maze Test (EPM)}

The elevated plus maze (EPM) was performed for studying the anxiety-like behaviour [30] [31]. The EPM apparatus consists of a "+" shaped elevated maze which has 2 "open" or uncovered arms and 2 "closed" or covered arms ( $5 \mathrm{~cm}$ width, $10 \mathrm{~cm}$ height of closed wall) connected by a $5 \times 5 \mathrm{~cm}^{2}$ central area. This apparatus is raised $50 \mathrm{~cm}$ above the floor. The behaviour was video-tracked for 5 minutes and analyzed automatically using the ANYmaze ${ }^{\mathrm{TM}}$ Software. The maze was cleaned using doctor's alcohol after every individual mouse tested. The time spent in open arms, closed arms as well as the central area was used to compare the anxiety levels. Number of entries to the open and closed arms was also considered to quantify the anxiety-like behaviour. Entries into an area were considered only when the mouse placed all four paws in that area.

\subsection{Forced Swim Test (FST)}

Forced swim test (FST) was performed to test the depressive phenotype [32]. Briefly, the mice were forced to swim in a vertical cylindrical jar (30 cm in height and $10 \mathrm{~cm}$ in diameter) filled with water $\left(25^{\circ} \mathrm{C}-27^{\circ} \mathrm{C}\right.$ ) to a depth of $20 \mathrm{~cm}$. Every mouse was placed gently in this apparatus and observed for $6 \mathrm{~min}$. After each test session, the mice were dried with a cloth, and placed back in the home cage. The scored parameters include latency to first immobility, number of bouts of immobility over the last 4 min of a total 6 min observation time and number of fecal boli.

\subsection{Morris Water Maze Test (MWM)}

To evaluate the effect of chronic dietary administration of 2-DG on learning (acquisition) and memory (recall), another set of experimental animals was employed to test spatial learning and memory using Morris water maze [33] [34]. Briefly, the apparatus consisted of a circular tank (diameter $120 \mathrm{~cm}$, depth $100 \mathrm{~cm}$ ) was filled with water $\left(25^{\circ} \mathrm{C}-27^{\circ} \mathrm{C}\right)$ to a depth of $50 \mathrm{~cm}$. Nontoxic water soluble paint was added to make the water opaque so as to hide the goal platform $0.5-0.8 \mathrm{~cm}$ below the water. The pool was divided in 4 imaginary quadrants designated as NW, NE, SE, SW, along the north (N)-south (S) axis and east (E)-west (W) axis, using the automated tracking software ANYmaze ${ }^{\mathrm{TM}}$. Intra-maze visual cues were provided, while the immovable objects like windows and observer seat served as the extra maze cues. Utilizing these cues, the mice were made to learn the spatial position of the hidden platform by performing 20 learning trials over 5 days with a maximum of 4 trials per day. A trial was considered to be completed after the mice was released facing the pool wall from each quadrant's start position. The mice were allowed to swim and find the hidden platform for a maximum latency of 120 
s. If any mouse was unable to find the platform, it was gently guided to the platform and allowed to acquire the spatial cues for 15 seconds before being removed, dried and kept back in its home cage. The latency to reach the platform as well as the distance travelled until entry to target was recorded and compared to observe any differences in learning abilities. After the completion of the learning trials, a probe trial was performed 24 hours after the last acquisition trial to test the long term spatial memory functions. The platform was removed and the animals were allowed to swim freely for $30 \mathrm{~s}$ for 4 releases. The latency to the first entry to target, number of entries to the target and the entries into the target quadrant were compared to visualize long term memory functions.

\subsection{Novel Object Recognition Test (NORT)}

This paradigm was carried out in the OFT arena as previously described [35] [36]. Briefly, two separate trials were carried out, the first acquisition trial, to familiarize the mice with two similar objects and subsequently, a second one, probe trial, where one familiar object was replaced with a novel object for testing the recognition memory. The box and the objects were properly cleaned after every test trial period to remove the olfactory cues from the previous mouse. The objects used in this test were plastic toys of different shapes. Exploration of an object was considered when the mice started sniffing the object from a maximum distance of $1 \mathrm{~cm}$ or less. Climbing and sitting on the object were not included in the exploratory activity. The time spent by the mice in exploring the novel object versus the time spent exploring the familiar object gave a notion of the recognition memory. The training trial was conducted for $5 \mathrm{~min}$, and after the completion of this period, the mice were returned to their home cage for $10 \mathrm{~min}$. Subsequently, the probe trial was conducted for duration of 3 min, wherein one familiar object was replaced by a novel object, and the time spent in exploring each of the objects was recorded. Thus, the short-term memory dependent on the hippocampus was observed. The Discriminatory Index (DI) of mice was calculated as follows:

$$
\begin{aligned}
\mathrm{DI}= & {[(\text { Time spent exploring novel object } / \text { Total exploration time })} \\
& -(\text { Time spent exploring familiar object/Total exploration time })] \times 100
\end{aligned}
$$

The active exploration was only considered when both the forelimbs of the mouse were within the $1.5 \mathrm{~cm}$ circle around the objects, with the head facing the object or with the whiskers touching it.

\subsection{Grip Strength Test}

Grip strength was assessed as discussed earlier [37] using the grip strength meter system (Stoelting, USA). The test was performed to determine the neuro-muscular integrity in 2-DG fed or control mice. Mice were allowed to hold on to the grid with both forepaws (for 2-paw grip strength measurement) to obtain the maximum force applied when pulled away from the instrument. The force values obtained were tension values in gram-force (gF), consequently, the modulus of the numerical values was used to depict the graph. Each mouse was given 3 trials within the course of 2 minutes, and average values were used to represent the grip strength of an individual mouse.

\subsection{Statistical Analysis}

All the data were analyzed using GraphPad Prism (version 5.01). One-way ANOVA followed by Bonferroni’s post hoc test was used to assess the significance level. Statistical significance was taken at 95\% confidence intervals $(\mathrm{p} \leq 0.05)$.

\section{Results}

\subsection{Dietary 2-DG Increased Spontaneous Locomotor Activity, While Decreasing Stress and Anxiety}

We first examined the locomotor activity along with stress and anxiety-like behaviour of mice fed with 2-DG using the open field (OF) test. The 2-DG fed mice showed increased locomotor activity as compared to the control mice, as evidenced by the number of squares crossed (grid lines crossed during 5 min), an indicator of hori- 
zontal activity. The number of grid lines crossed were significantly higher in animals fed on 2-DG with 75 and 63 grid lines in $0.2 \%$ and $0.4 \%$ 2-DG groups respectively (0.2\% 2-DG; $\mathrm{p}<0.01,0.4 \% 2$-DG; $\mathrm{p}<0.05$ ) compared to 22 in the control group (Figure 1(A)). The rearing evaluated as a measure of vertical activity in the open field showed no significant difference between 2-DG fed mice as compared to the control mice (Figure 1(B)). The 2-DG fed mice reared 26 and 21 times at $0.2 \%(p=0.4)$ and $0.4 \%(p=0.66)$ doses respectively in comparison to 21 times in the controls.

The anxiety-like behaviour was evaluated by measuring the time taken to enter the center and time spent in the center of the open field box. The latency to center showed moderate reduction in both the 2-DG fed groups (Figure 1(C); 235 sec in 0.2\%; $\mathrm{p}=0.24$ and $216 \mathrm{sec}$ in $0.4 \%$; $\mathrm{p}=0.18$ ) as compared to control (279 sec), which was not statistically significant. The average time spent in the center was also not significantly altered in 2-DG fed mice (Figure $1(\mathrm{D})$ ), with 3 and 8 sec in the $0.2 \%(\mathrm{p}=0.18)$ and $0.4 \%(\mathrm{p}=0.34) 2$-DG groups compared to 1 sec in the unfed mice. The 2-DG fed animals were relatively less anxious as they spent more time in the center in comparison to the control animals. Interestingly, the number of mice entering the center was higher in 2-DG treated groups (4/6 in $0.2 \%$ 2-DG and 3/6 in $0.4 \%$ 2-DG) compared to the untreated group (1/6; data not shown). The 2-DG fed mice also showed enhanced grooming at both the 2-DG doses; 1.33 in $0.2 \%(\mathrm{p}=0.39)$ and 1.83 in $0.4 \%$ ( $<0.05$; statistically significant) as compared to 0.83 in control (Figure $1(\mathrm{E})$ ). The defecation rate was
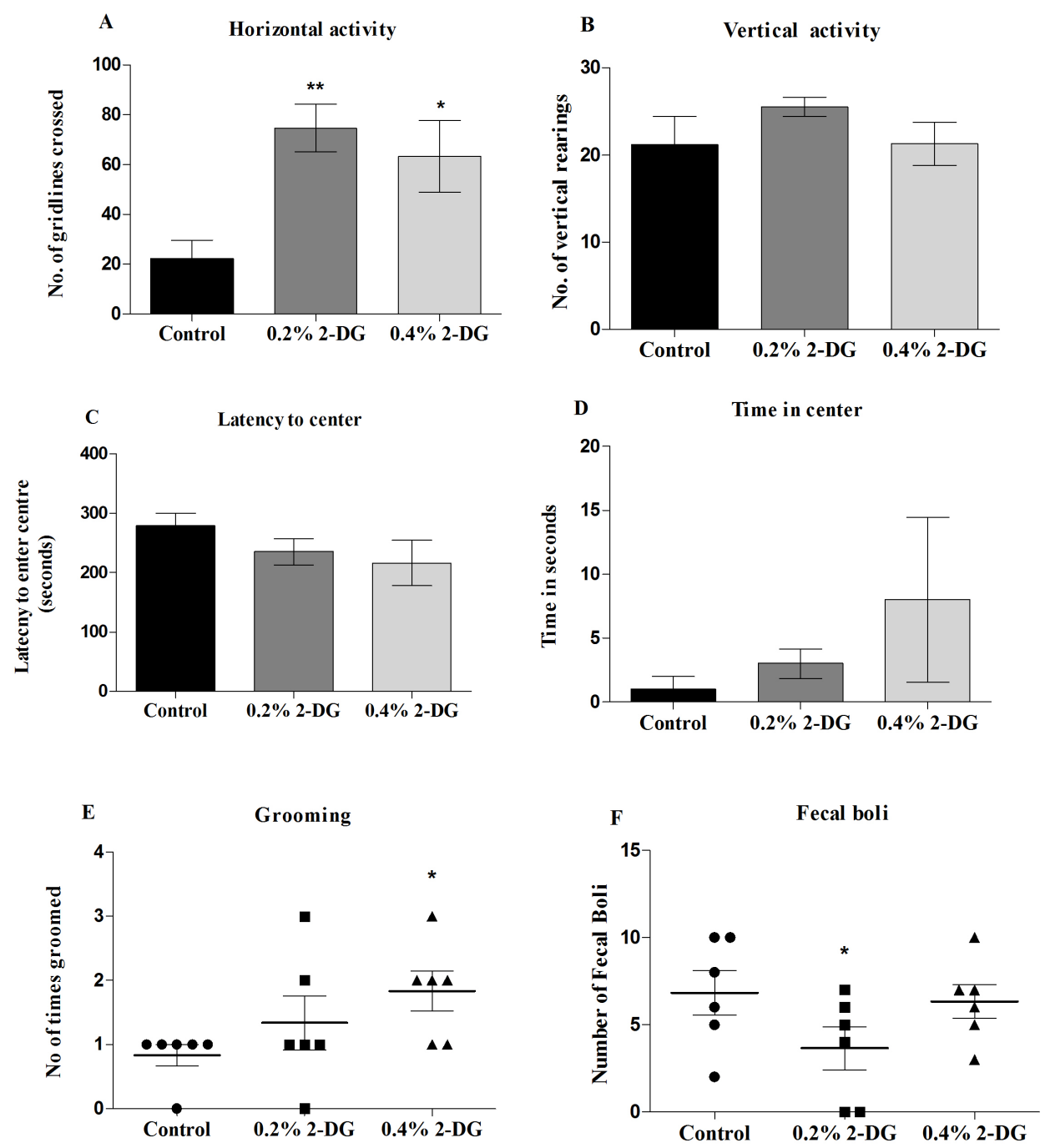

Figure 1. Locomotor activity and anxiety-like behaviour in the open field test (OFT). (A) Horizontal activity; (B) Vertical activity; (C) Latency to center; (D) Time in center; (E) Grooming; (F) Fecal boli. Values presented are mean \pm SE; One-way ANOVA, ${ }^{*} \mathrm{p}<0.05,{ }^{* *} \mathrm{p}<0.01$. 
significantly lower in 0.2\% 2-DG group (3.6; $\mathrm{p}<0.5$ ) and comparable to control (6.8), in 0.4\% 2-DG group (6.3; $\mathrm{p}=0.74$ ) (Figure $1(\mathrm{~F})$ ). Increased grooming and reduced fecal boli suggested that chronic dietary administration of 2-DG employed here does not cause any stress or anxiety-like behaviour.

We also employed the EPM test to further confirm the changes related to stress and anxiety-like behaviour exhibited in the OF test. The latency to enter the center (Figure 2(A)) with two-side open arms was measured and found to be reduced in the 2-DG groups in a dose dependent manner, which was however not-significant; $34.5 \mathrm{sec}$ in $0.2 \%(\mathrm{p}=0.087)$ and $22.2 \mathrm{sec}$ in $0.4 \%(\mathrm{p}=0.51) 2-\mathrm{DG}$ groups against $38.8 \mathrm{sec}$ in the control. Also, the 2-DG fed mice spent slightly longer time in the center (Figure 2(B); 58.8 and 60.8 sec in $0.2 \%$; $=0.46$ and $0.4 \%$; $=0.4$, 2-DG respectively) compared to the control mice (50 sec). The fecal boli were counted during the EPM test and no significant differences were found between the groups (Figure 2(C); $\mathrm{p}=0.67$ ). These observations lend support to the proposition that the dietary 2-DG administration adopted in the present studies did not negatively affect the behaviour as revealed by OF and EPM tests. Indeed it marginally reduced both stress and anxiety-like behaviour.

\subsection{Dietary 2-DG Does Not Induce Depression Like Symptoms}

Forced swim test was performed to assess any signs of 2-DG induced depression in the mice. The latency to first immobility in the 2-DG mice was comparable to the untreated mice (Figure 3(A); 0.2\% 2-DG; $p=0.75$ and $0.4 \%$ 2-DG; $p=0.83$ ). Similarly, significant differences were also not observed in the bouts of immobility in the 2-DG fed animals (Figure 3(B); $p=0.4$ in $0.2 \%$ and $p=0.83$ in $0.4 \%$ 2-DG). 2-DG fed mice were immobile for slightly lesser period, with an average of $2.62 \mathrm{sec}$ in $0.2 \%$ 2-DG (p=0.6) and 2.0 sec in $0.4 \% 2-\mathrm{DG}(\mathrm{p}=0.3)$, as compared to $2.87 \mathrm{sec}$ in the control mice (Figure 3(C)).

\subsection{Dietary 2-DG Slightly Enhances Short-Term Memory}

Novel object recognition test (NORT) was performed to determine whether chronic dietary 2-DG administration has any adverse effect on short-term memory functions. The 2-DG fed mice exhibited decrease in the latency to novel object in the test phase; $56.6 \mathrm{sec}$ in control versus 37.4 and $32.2 \mathrm{sec}$ in $0.2 \%(\mathrm{p}=0.3)$ and $0.4 \%(\mathrm{p}=0.13)$

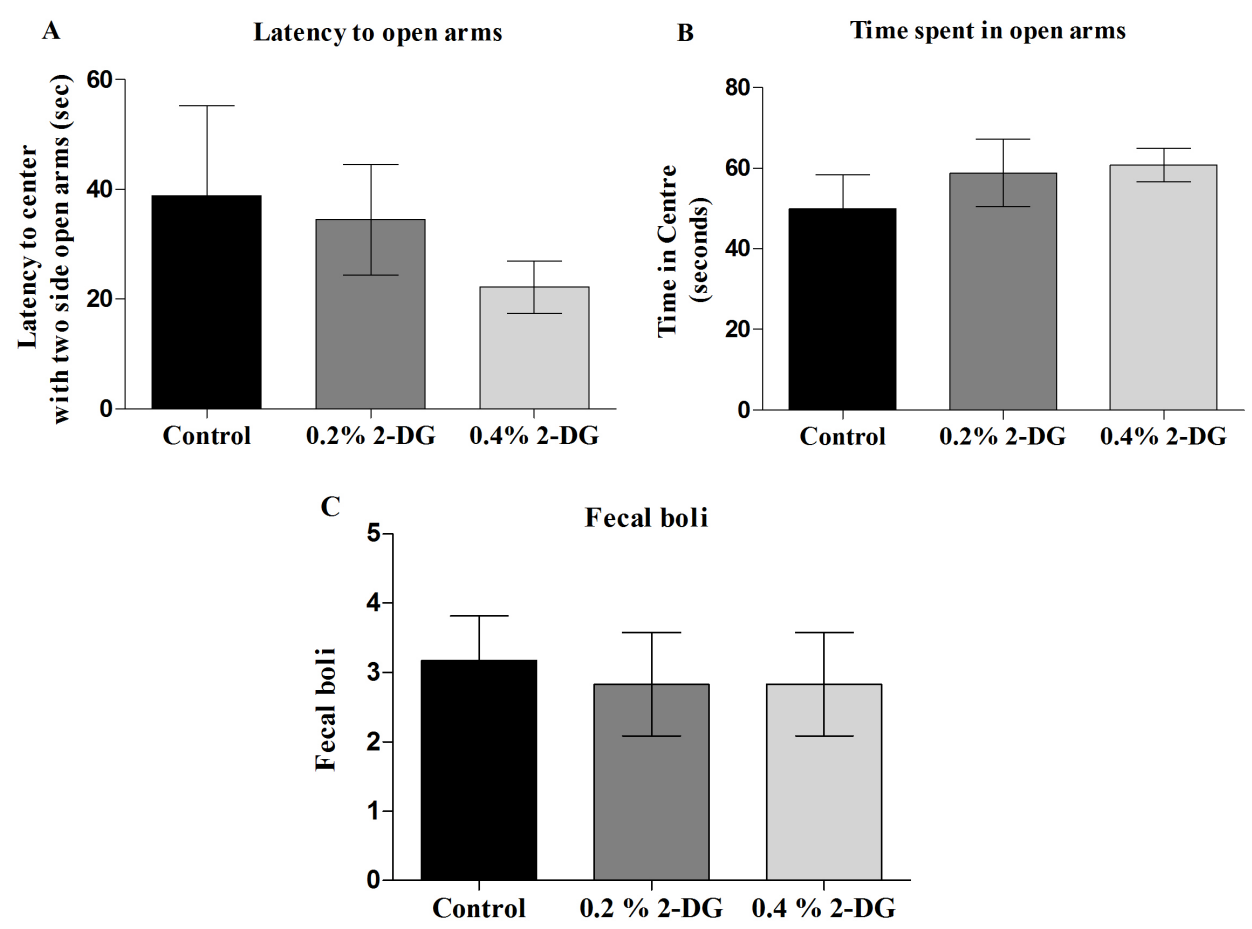

Figure 2. Locomotor activity and anxiety-like behaviour in the elevated plus maze (EPM). (A) Latency to open arms; (B) Time spent in open arms; (C) Fecal boli. Values are given as mean \pm SE; One-way ANOVA. 

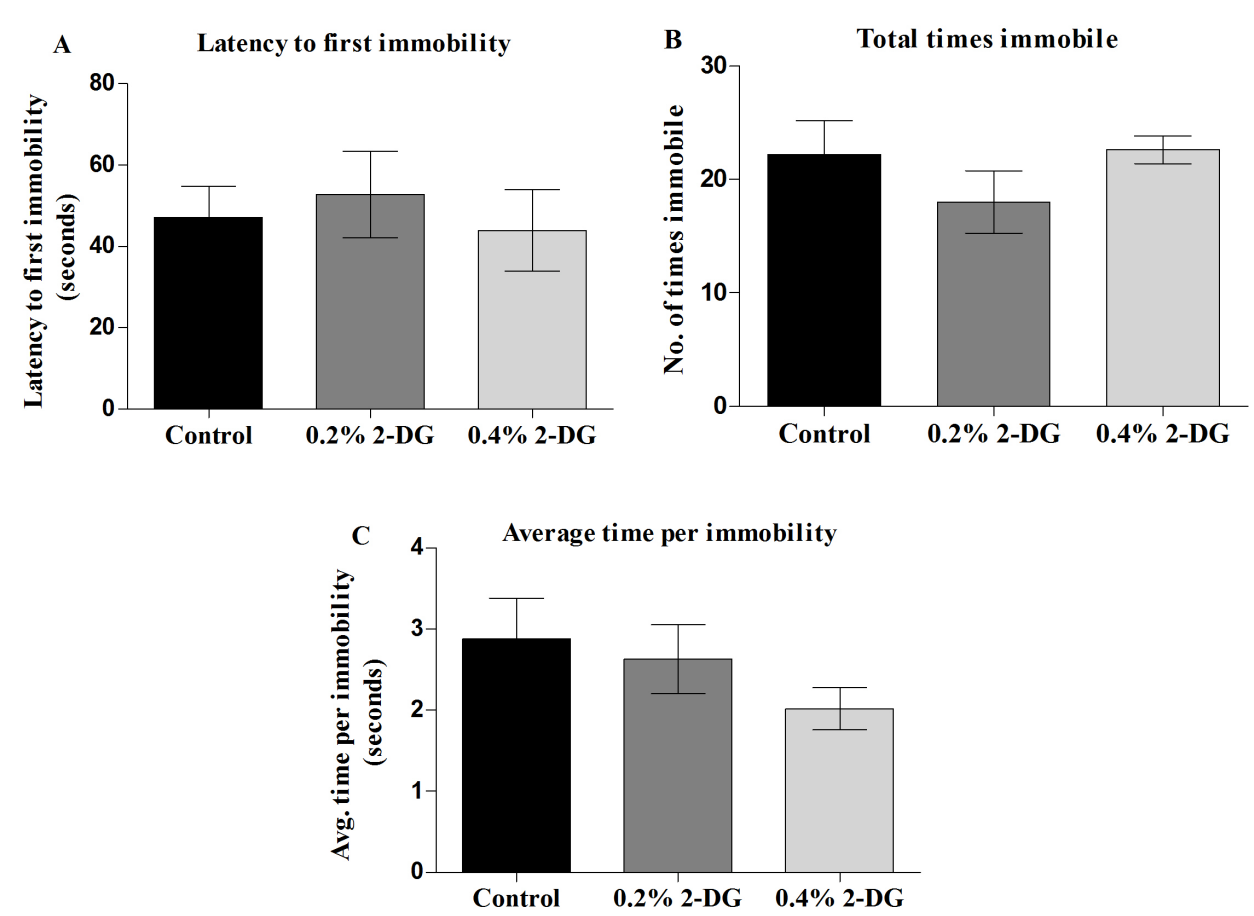

Figure 3. Forced swim test for depression like behaviour. (A) Latency to first immobility; (B) Total number of times immobile; (C) Average times per immobility. Values are given as mean \pm SE; One-way ANOVA.

2-DG (Figure 4(A)). These results indicate lack of any adverse effect, but a slightly enhanced short term memory function in mice administered with dietary 2-DG. At $0.2 \%$ 2-DG dose mice showed higher preference for novel object as suggested by the increased discriminatory index, while at $0.4 \%$ 2-DG it was similar to the unfed mice. These differences were statistically nonsignificant with discriminatory index (DI) of 0.34 and 0.17 in $0.2 \%$ $(p=0.52)$ and $0.4 \%(p=0.93)$ 2-DG respectively compared to 0.18 of control group (Figure 4(B)). These observations suggested that dietary 2-DG under the present experimental conditions did not hamper the cognition; instead it perhaps marginally improved as $0.2 \%$ 2-DG fed mice spent relatively more time exploring and interacting with the novel object than the familiar object.

\subsection{Dietary 2-DG Preserved the Hippocampus Based Long Term Learning and Memory Functions}

Morris water maze (MWM) test was employed for evaluating the effects of 2-DG on long term cognition. Firstly, we observed that the total distance travelled by the 2-DG treated and untreated mice was comparable throughout the learning phase (Figure 5(A)). Control and 2-DG fed mice both showed a similar decrease in the latency to the platform in the learning trials on all the consecutive days suggesting that 2-DG did not compromise or alter the learning task (Figure 5(B)). On the final day of the acquisition trial (learning phase), mice from the 2-DG groups took less time to reach the platform than the mice from the untreated group (Figure 5(B); $16.1 \mathrm{sec}$ in control versus $8.8 \mathrm{sec}$ in $0.2 \%$ 2-DG and $11.3 \mathrm{sec}$ in $0.4 \% 2-\mathrm{DG}$; over all $\mathrm{p}=0.04$ ). Although the differences observed were not statistically significant, they were indicative of preserved normal learning functions. 2-DG treated animals did show a consistent decreasing trend in the total distance travelled to reach the platform each day of trial similar to untreated groups with no differences in their swimming performance, suggesting that the learning processes were not affected by chronic 2-DG treatment.

For the probe trial, the platform was removed and the number of times the mice crossed the target probe (platform) during 30 sec was measured (Figure 5(C)). 2-DG fed mice showed a slightly better memory as indicated by relatively higher number of target platform crossings as compared to the control mice, although the differences were statistically nonsignificant ( 2.6 and 3 in $0.2 \%$; $p=0.52$ and $0.4 \% 2$-DG; $p=0.08$ vs 1.6 in control). Similarly, the number of entries to the target quadrant did not significantly differ between the 2-DG fed 

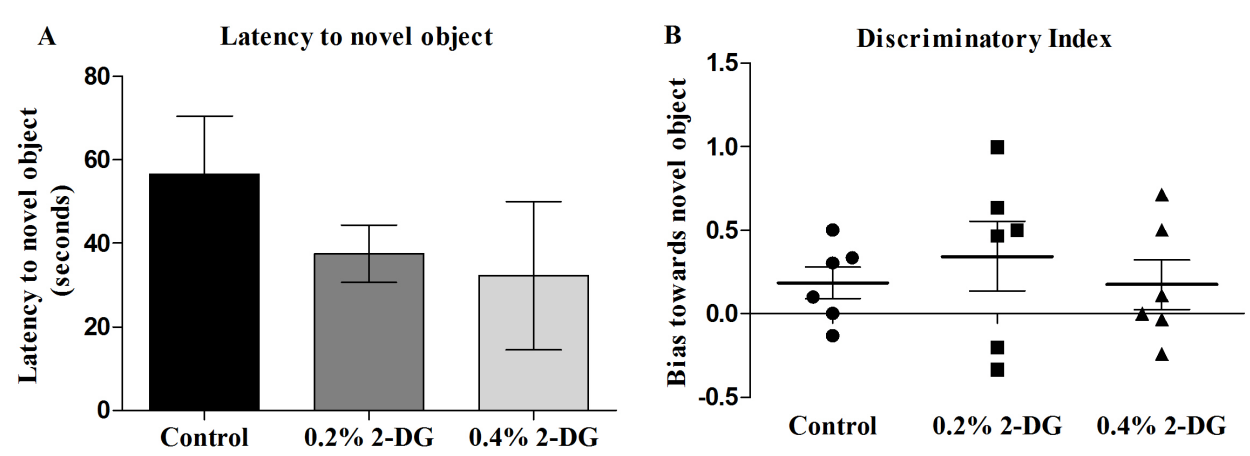

Figure 4. The novel object recognition memory test (NORT) was performed to assess the effect of dietary 2-DG on learning and memory process. (A) Latency to novel object; (B) Discrimination indices. Values are given as mean \pm SE; Statistical analysis by One-way ANOVA.
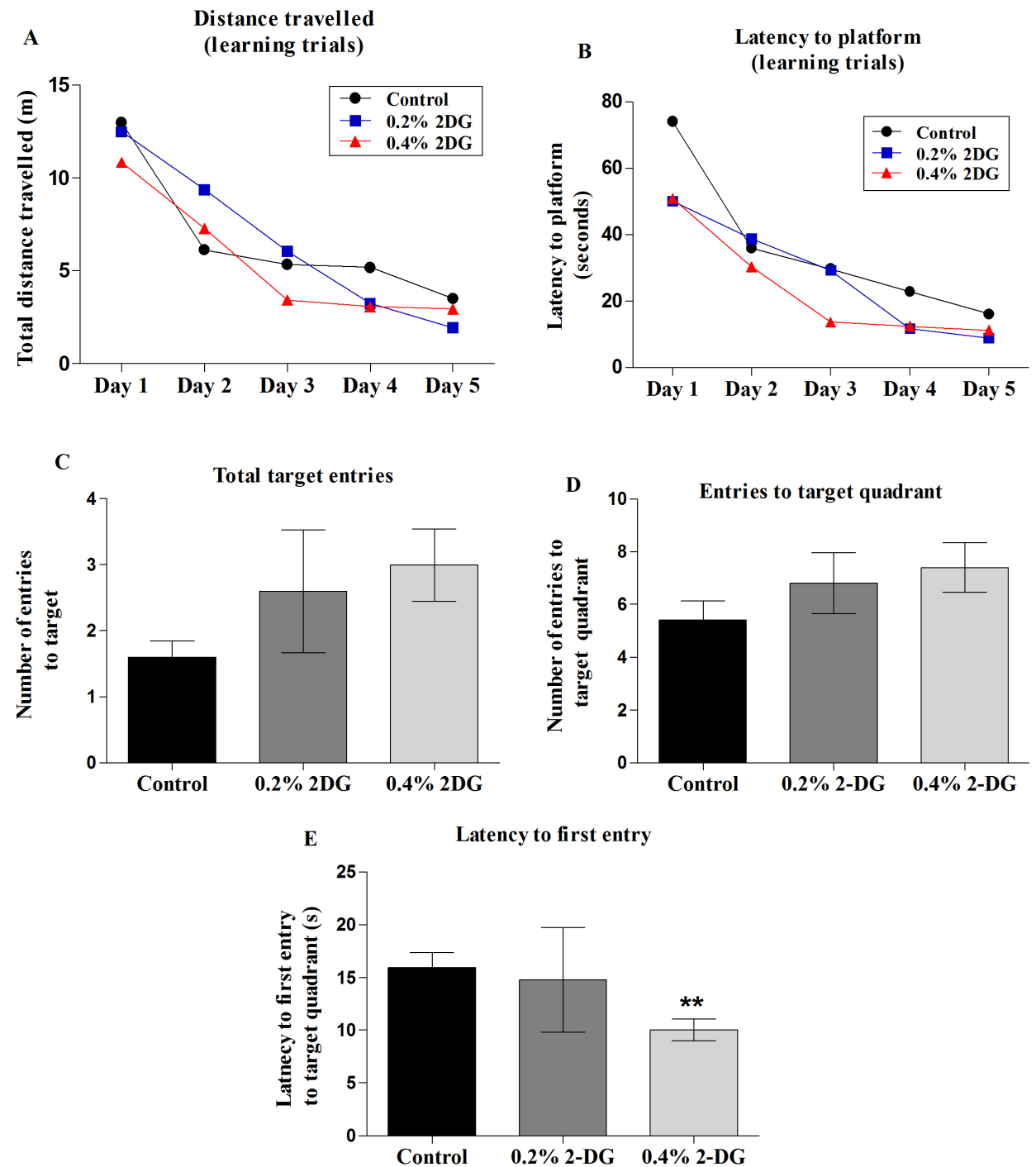

Figure 5. Morris water maze (MWM) test was performed to assess the effect of dietary 2-DG on long term memory process. (A) Total distance travelled; (B) Latency to platform during acquisition trial; (C) Total number of entries to the target; (D) Number of entries to target quadrant; (E) Latency to first entry to target quadrant during probe trial. Values are given as mean \pm SE; Statistical analysis by One-way ANOVA. 
and the unfed mice (Figure 5(D)). The number of entries to the target quadrant was 6.8 in $0.2 \% 2$-DG (p = 0.4) and 7.4 in $0.4 \%$ 2-DG $(\mathrm{p}=0.19)$ in comparison to 5.4 in the control mice. Further, the 2-DG fed mice took less time to reach the target quadrant. The latency was decreased to $14.7 \mathrm{sec}$ in $0.2 \%(\mathrm{p}=0.69)$ and significantly reduced (statistically) to $10 \mathrm{sec}$ in $0.4 \%$ 2-DG $(\mathrm{p}=0.007$ ) as compared to $16 \mathrm{sec}$ in unfed mice (Figure $5(\mathrm{E})$ ). These data demonstrated that memory, as assessed by probe trial was not adversely affected by chronic dietary administration of 2-DG.

\subsection{Neuro-Muscular Functional Integrity Was Not Compromised by 2-DG}

Glucose metabolism is very important for the muscle cells and 2-DG being a glycolytic inhibitor and administered for chronic period, it was considered worthwhile to evaluate the effects of chronic 2-DG administration on the neuro-muscular integrity. We performed grip strength test and recorded 2-paw (forepaw) grip strength measurements. Under the present experimental conditions, 2-DG had no apparent effect on the grip strength (Figure 6).

\section{Discussion}

In the recent times, there has been a growing interest in the identification of potent ERMs for cancer preventive, anti-aging and age-associated diseases. Since quality of life is an important consideration in cancer therapy and prevention, it is considered important to evaluate the effects of ERMs on the physiological status as well as functional aspects, for safety and successful implementation. In the present studies, we evaluated the effects of 2-DG (given in daily drinking water at doses $0.2 \%$ and $0.4 \% \mathrm{w} / \mathrm{v}$ ), a potential ERM, on behaviour, under condition that was earlier shown to inhibit implanted tumor growth and induced tumorigenesis in mice [25] [26]. We found no evidence of any adverse effects of 2-DG on the behavioural performances observed at the end of 3 months of administration that has been shown to significantly impair tumorigenesis. Besides, inhibition of tumorigenesis, administration of 2-DG has been shown to be significantly beneficial in reducing ageing and age related other neurodegenerative disorders such as Huntington's disease, Parkinson's disease, Alzheimer's disease [6] [12] [38]-[40].

Several studies investigating the overall effects of chronic DER suggest a reduction in the age related decline in spontaneous motor activity and anxiety-like behaviour [41]-[43] along with a decrease in the age-related cognitive impairment [44]. Contrary to this, certain studies have shown that DER results in depressive and/or anxiety-like behavioural pattern as a consequence of deregulated cerebral serotonin neurotransmission axis/network [45]. Chronic DER designed to reduce body weight causes eating disorders and depressive behaviour [46], while lifelong DER failed to prevent age related impairment in spatial learning [47]. However, the present study demonstrates that chronic dietary use of metabolic modifier 2-DG as an alternate to DER provides an advantage over the conventional DER as it does not cause/aggravate the stress and anxiety-like behaviour, locomotor activity and cognition disturbances at cancer preventive doses, unlike in the case of chronic DER studies.

Dietary feeding of 2-DG at the doses used here posed no negative effect on the exploratory activity. On the contrary, 2-DG fed mice showed increased spontaneous locomotor activity as reflected by the increased horizontal activity (number of grid lines crossed) in OFT. Also, the vertical movement in 2-DG groups was similar to that of the untreated mice. Earlier, studies have shown that rats fed with 2-DG in diet have increased dopamine related locomotor responses [48]. 2-DG fed mice showed slightly reduced latency to enter the center with

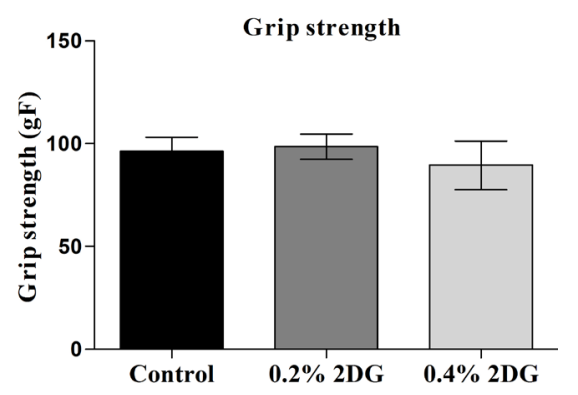

Figure 6. Grip strength test for neuro-muscular integrity. Values are given as mean $\pm \mathrm{SE}$. 
relatively more time spent in center compared to control animals, suggesting that 2-DG did not induce more anxiety. Furthermore, the 2-DG fed mice had increased grooming behaviour with decreased defecation rate indicating slight reduction in basal test condition related stress and anxiety. These findings were further supported by results in the EPM, where the performance of the 2-DG fed mice was not compromised.

In the FST, immobility time was slightly shortened in 2-DG fed mice, indicating reduced depressive-like behaviour. We also demonstrated that mice fed with 2-DG display slightly improved cognitive functions (short term learning and memory), as evidenced by slightly increased discriminatory index in NORT. Most importantly, no detrimental effects of chronic 2-DG feeding on cognition was observed. In addition to this, mice subjected to dietary 2-DG had a slightly improved learning performance and spatial memory in the water maze test. These observations imply that extended administration of 2-DG as a component of the diet does not hamper the synaptic plasticity in the hippocampus, which plays a central role in long term learning and memory processes [33] [49]. Neuroprotective effects of 2-DG has been demonstrated by its effectiveness against convulsions and epileptic seizures in rat model, with anticonvulsant and antiepileptic actions related to the modulation of glucose metabolism [27]. In Parkinson's disease model of mice, 2-DG has been shown to induce faster recovery in behavioural disturbances caused by the neurotoxin, 1-methyl-4-phenyl-1,2,3,6-tetrahydropyridine (MPTP) [38].

Our findings suggest that dietary administration of 2-DG at doses previously shown to be tumor inhibitory [25] is well-tolerated, in terms of coping up with stress and anxiety-like behaviour, locomotor activity and cognition. The uncompromised and or slightly improved behavioural performances observed in this study may likely involve better glucoregulation (reduced blood glucose and serum insulin levels) and reduced cellular glucose availability along with 2-DG uptake posing mild metabolic stress [25]. This metabolic stress posed by 2-DG is known to cause metabolic adaptation such as ketosis [39], thereby reducing the oxidative stress and reduced oxidative damage to tissues and organs and importantly brain. 2-DG induced ketosis may at least in part account for the lack of any adverse effects on CNS/behaviour. Elevated levels of circulating ketones are thought to underlie the therapeutic effects by bypassing cytoplasmic glycolysis and provide energy directly through the Krebs cycle [50] [51]. On the contrary, chronic consumption of sucrose in murine model of amyloid deposition has been shown to induce glucose intolerance, insulin resistance and deterioration of cognitive functions with enhanced $\mathrm{A} \beta$ deposits [52].

It is pertinent to mention that in the present study, all behaviour parameters were tested at 6 months of age (i.e. after 3 months of chronic dietary 2-DG administration) and not in aged mice. Therefore, these results cannot be extrapolated for age related impairments in the cognition. Nevertheless, it is expected that dietary 2-DG would provide similar or improved behavioural performance in aged mice as well. This report does not include any molecular level study, since the aim of our study was to evaluate the safety aspects of chronic dietary 2-DG administration, further studies are required to examine the effects on age-related behavioural consequences with insight on the underlying mechanisms.

\section{Conclusion}

There is a considerable amount of interest in using 2-DG alone or in combination with radio- or chemotherapy for the treatment of various cancers. Feasibility of administering 2-DG in combination with hypofractionated irradiation of the tumor has been shown in malignant glioma patients without acute or late toxicity [21]. Since, effective systemic 2-DG levels can be achieved upon oral administration, considerable levels can be attained even in the brain as it can cross the blood brain barrier similar to glucose [53]. Since brain mainly utilizes glucose for energy, CNS toxicity and functional disturbances could be causes of concern and limitations in using 2-DG. However, findings of the present studies provide evidence that the use of 2-DG in a chronic dietary regimen is a safe anti-tumor strategy at $0.2 \%$ and $0.4 \% 2-\mathrm{DG}(\mathrm{w} / \mathrm{v})$ doses.

\section{Acknowledgements}

Pharmacological grade 2-deoxy-D-glucose (2-DG) was generously provided by Dr Reddy's Laboratories Ltd. (Hyderabad, India). We thank Dr. R.P. Tripathi, Director INMAS for constant support and encouragement.

\section{References}

[1] Ingram, D.K., Zhu, M., Mamczarz, J., Zou, S., Lane, M.A., Roth, G.S. and DeCabo, R. (2006) Calorie Restriction Mi- 
metics: An Emerging Research Field. Aging Cell, 5, 97-108. http://dx.doi.org/10.1111/j.1474-9726.2006.00202.x

[2] Heilbronn, L.K. and Ravussin, E. (2003) Calorie Restriction and Aging: Review of the Literature and Implications for Studies in Humans. The American Journal of Clinical Nutrition, 78, 361-369.

[3] Barger, J.L., Kayo, T., Vann, J.M., Arias, E.B., Wang, J., Hacker, T.A., Wang, Y., et al. (2008) A Low Dose of Dietary Resveratrol Partially Mimics Caloric Restriction and Retards Aging Parameters in Mice. PLoS ONE, 3, e2264. http://dx.doi.org/10.1371/journal.pone.0002264

[4] Lin, J.N., Lin, V., Rau, K.M., Shieh, P.C., Kuo, D.H., Shieh, J.C., Chen, W.J., et al. (2010) Resveratrol Modulates Tumor Cell Proliferation and Protein Translation via SIRT1-Dependent AMPK Activation. Journal of Agricultural and Food Chemistry, 58, 1584-1592. http://dx.doi.org/10.1021/jf9035782

[5] Thompson, H., Zhu, Z., Thompson, M., Jiang, W. and McGinley, J. (2011) Metformin as an Energy Restriction Mimetic Agent for Breast Cancer Prevention. Journal of Carcinogenesis, 10, 17. http://dx.doi.org/10.4103/1477-3163.83043

[6] Lane, M.A., Ingram, D.K. and Roth, G.S. (1998) 2-Deoxy-D-Glucose Feeding in Rats Mimics Physiologic Effects of Calorie Restriction. Journal of Anti-Aging Medicine, 1, 327-337. http://dx.doi.org/10.1089/rej.1.1998.1.327

[7] Zhu, Z., Jiang, W., McGinley, J.N. and Thompson, H.J. (2005) 2-Deoxyglucose as an Energy Restriction Mimetic Agent: Effects on Mammary Carcinogenesis and on Mammary Tumor Cell Growth in Vitro. Cancer Research, 65, 7023-7030. http://dx.doi.org/10.1158/0008-5472.CAN-05-0453

[8] Warburg, O., Wind, F. and Negelein, E. (1927) The Metabolism of Tumors in the Body. The Journal of General Physiology, 8, 519-530. http://dx.doi.org/10.1085/jgp.8.6.519

[9] Warburg, O. (1956) On the Origin of Cancer Cells. Science, 123, 309-314. http://dx.doi.org/10.1126/science.123.3191.309

[10] Woodward, G.E. and Carmer, F.B. (1952) 2-Deoxy-D-Glucose as an Inhibitor of Anaerobic Glycolysis in Tumor Tissues. Journal of the Franklin Institute, 254, 259-260. http://dx.doi.org/10.1016/0016-0032(52)90482-1

[11] Wan, R., Camandola, S. and Mattson, M.P. (2003) Intermittent Fasting and Dietary Supplementation with 2-Deoxy-DGlucose Improve Functional and Metabolic Cardiovascular Risk Factors in Rats. FASEB Journal: Official Publication of the Federation of American Societies for Experimental Biology, 17, 1133-1134. http://dx.doi.org/10.1096/fj.02-0996fje

[12] Wan, R., Camandola, S. and Mattson, M.P. (2004) Dietary Supplementation with 2-Deoxy-D-Glucose Improves Cardiovascular and Neuroendocrine Stress Adaptation in Rats. American Journal of Physiology-Heart and Circulatory Physiology, 287, H1186-H1193. http://dx.doi.org/10.1152/ajpheart.00932.2003

[13] Pelicano, H., Martin, D.S., Xu, R.H.H. and Huang, P. (2006) Glycolysis Inhibition for Anticancer Treatment. Oncogene, 25, 4633-4646. http://dx.doi.org/10.1038/sj.onc.1209597

[14] Dwarakanath, B. and Jain, V. (2009) Targeting Glucose Metabolism with 2-Deoxy-D-Glucose for Improving Cancer Therapy. Future Oncology, 5, 581-585. http://dx.doi.org/10.2217/fon.09.44

[15] Gupta, S., Farooque, A., Adhikari, J.S., Singh, S. and Dwarakanath, B.S. (2009) Enhancement of Radiation and Chemotherapeutic Drug Responses by 2-Deoxy-D-Glucose in Animal Tumors. Journal of Cancer Research and Therapeutics, 5, S16-S20. http://dx.doi.org/10.4103/0973-1482.55135

[16] Singh, D., Banerji, A.K., Dwarakanath, B.S., Tripathi, R.P., Gupta, J.P., Mathew, T.L., et al. (2005) Optimizing Cancer Radiotherapy with 2-Deoxy-D-Glucose: Dose Escalation Studies in Patients with Glioblastoma Multiforme. Strahlentherapie und Onkologie, 181, 507-514. http://dx.doi.org/10.1007/s00066-005-1320-z

[17] Pina, Y., Houston, S.K., Murray, T.G., Boutrid, H., Celdran, M., Feuer, W., Shi, W., et al. (2010) Focal, Periocular Delivery of 2-Deoxy-D-Glucose as Adjuvant to Chemotherapy for Treatment of Advanced Retinoblastoma. Investigative Ophthalmology and Visual Science, 51, 6149-6156. http://dx.doi.org/10.1167/iovs.09-5033

[18] Raez, L., Papadopoulos, K., Ricart, A., Chiorean, E., DiPaola, R., Stein, M., Lima, C., et al. (2012) A Phase I DoseEscalation Trial of 2-Deoxy-D-Glucose Alone or Combined with Docetaxel in Patients with Advanced Solid Tumors. Cancer Chemotherapy and Pharmacology, 71, 523-530. http://dx.doi.org/10.1007/s00280-012-2045-1

[19] Farooque, A., Singh, N., Adhikari, J.S., Afrin, F. and Dwarakanath, B.S.R. (2014) Enhanced Antitumor Immunity Contributes to the Radio-Sensitization of Ehrlich Ascites Tumor by the Glycolytic Inhibitor 2-Deoxy-D-Glucose in Mice. PLoS ONE, 9, e108131. http://dx.doi.org/10.1371/journal.pone.0108131

[20] Stein, M., Lin, H., Jeyamohan, C., Dvorzhinski, D., Gounder, M., Bray, K., Eddy, S., et al. (2010) Targeting Tumor Metabolism with 2-Deoxyglucose in Patients with Castrate-Resistant Prostate Cancer and Advanced Malignancies. Prostate, 70, 1388-1394. http://dx.doi.org/10.1002/pros.21172

[21] Mohanti, B.K., Rath, G.K., Anantha, N., Kannan, V., Das, B.S., Chandramouli, B.A., Banerjee, A.K., et al. (1996) Improving Cancer Radiotherapy with 2-Deoxy-D-Glucose: Phase I/II Clinical Trials on Human Cerebral Gliomas. International Journal of Radiation Oncology, Biology, Physics, 35, 103-111. 
http://dx.doi.org/10.1016/S0360-3016(96)85017-6

[22] Dwarakanath, B.S., Singh, D., Banerji, A.K., Sarin, R., Venkataramana, N.K., Jalali, R., Vishwanath, P.N., et al. (2009) Clinical Studies for Improving Radiotherapy with 2-Deoxy-D-Glucose: Present Status and Future Prospects. Journal of Cancer Research and Therapeutics, 5, S21-S26. http://dx.doi.org/10.4103/0973-1482.55136

[23] Goldstein, D.S., Breier, A., Wolkowitz, O.M. and Pickar, D. (1992) Plasma Levels of Catecholamines and Corticotrophin during Acute Glucopenia Induced by 2-Deoxy-D-Glucose in Normal Man. Clinical Autonomic Research, 2, 359366. http://dx.doi.org/10.1007/BF01831392

[24] Lin, X., Zhang, F., Bradbury, C.M., Kaushal, A., Li, L., Spitz, D.R., Aft, R.L., et al. (2003) 2-Deoxy-D-GlucoseInduced Cytotoxicity and Radiosensitization in Tumor Cells is Mediated via Disruptions in Thiol Metabolism. Cancer Research, 63, 3413-3417.

[25] Singh, S., Pandey, S., Bhatt, A.N., Chaudhary, R., Bhuria, V., Kalra, N., Soni, R., et al. (2015) Chronic Dietary Administration of the Glycolytic Inhibitor 2-Deoxy-D-Glucose (2-DG) Inhibits the Growth of Implanted Ehrlich's Ascites Tumor in Mice. PLOS ONE, 10, e0132089. http://dx.doi.org/10.1371/journal.pone.0132089

[26] Singh, S., Oberoi, R., Khanna, S., Bhatt, A., Saluja, D. and Dwarakanath, B. (2012) Energy Restriction Caused by the Glycolytic Inhibitor, 2-Deoxy-D-Glucose Inhibits Chemical Induced Carcinogenesis in Mice. Proceedings of the 103rd Annual Meeting of the American Association for Cancer Research, Chicago, March 31-April 42012.

[27] Stafstrom, C., Ockuly, J., Murphree, L., Valley, M., Roopra, A. and Sutula, T. (2009) Anticonvulsant and Antiepileptic Actions of 2-Deoxy-D-Glucose in Epilepsy Models. Annals of Neurology, 65, 435-447. http://dx.doi.org/10.1002/ana.21603

[28] Ockuly, J., Gielissen, J., Levenick, C., Zeal, C., Groble, K., Munsey, K., Sutula, T., et al. (2012) Behavioral, Cognitive, and Safety Profile of 2-Deoxy-2-Glucose (2DG) in Adult Rats. Epilepsy Research, 101, 246-252. http://dx.doi.org/10.1016/j.eplepsyres.2012.04.012

[29] Eikelis, N. and Buuse, V.M. (2000) Cardiovascular Responses to Open-Field Stress in Rats: Sex Differences and Effects of Gonadal Hormones. Stress, 3, 319-334. http://dx.doi.org/10.3109/10253890009001137

[30] Carola, V., D’Olimpio, F., Brunamonti, E. and Mangia, F. (2002) Evaluation of the Elevated Plus-Maze and OpenField Tests for the Assessment of Anxiety-Related Behaviour in Inbred Mice. Behavioural Brain Research, 134, 49-57. http://dx.doi.org/10.1016/S0166-4328(01)00452-1

[31] Dawson, G.R. and Tricklebank, M.D. (1995) Use of the Elevated Plus Maze in the Search for Novel Anxiolytic Agents. Trends in Pharmacological Sciences, 16, 33-36. http://dx.doi.org/10.1016/S0165-6147(00)88973-7

[32] Porsolt, R.D., Pichon, L.M. and Jalfre, M.L. (1977) Depression: A New Animal Model Sensitive to Antidepressant Treatments. Nature, 266, 730-732. http://dx.doi.org/10.1038/266730a0

[33] Morris, R., Garrud, P., Rawlins, J. and O’Keefe, J. (1982) Place Navigation Impaired in Rats with Hippocampal Lesions. Nature, 297, 681-683. http://dx.doi.org/10.1038/297681a0

[34] Vorhees, C. and Williams, M. (2006) Morris Water Maze: Procedures for Assessing Spatial and Related Forms of Learning and Memory. Nature Protocols, 1, 848-858. http://dx.doi.org/10.1038/nprot.2006.116

[35] Ennaceur, A. and Delacour, J. (1988) A New One-Trial Test for Neurobiological Studies of Memory in Rats.1: Behavioral Data. Behavioural Brain Research, 37, 47-59. http://dx.doi.org/10.1016/0166-4328(88)90157-X

[36] Bevins, R.A. and Besheer, J. (2006) Object Recognition in Rats and Mice: A One-Trial Non-Matching-to-Sample Learning Task to Study Recognition Memory. Nature Protocols, 1, 1306-1311. http://dx.doi.org/10.1038/nprot.2006.205

[37] Kehl, L.J., Trempe, T.M. and Hargreaves, K.M. (2000) A New Animal Model for Assessing Mechanisms and Management of Muscle Hyperalgesia. Pain, 85, 333-343. http://dx.doi.org/10.1016/S0304-3959(99)00282-1

[38] Duan, W. and Mattson, M.P. (1999) Dietary Restriction and 2-Deoxyglucose Administration Improve Behavioral Outcome and Reduce Degeneration of Dopaminergic Neurons in Models of Parkinson's Disease. Journal of Neuroscience Research, 57, 195-206. http://dx.doi.org/10.1002/(SICI)1097-4547(19990715)57:2<195::AID-JNR5>3.0.CO;2-P

[39] Yao, J., Chen, S., Mao, Z., Cadenas, E. and Brinton, R. (2011) 2-Deoxy-D-Glucose Treatment Induces Ketogenesis, Sustains Mitochondrial Function, and Reduces Pathology in Female Mouse Model of Alzheimer's Disease. PLoS ONE, 6, e21788. http://dx.doi.org/10.1371/journal.pone.0021788

[40] Wei, J., Cohen, D.M. and Quast, M.J. (2003) Effects of 2-Deoxy-D-Glucose on Focal Cerebral Ischemia in Hyperglycemic Rats. Journal of Cerebral Blood Flow and Metabolism, 23, 556-564. http://dx.doi.org/10.1097/01.WCB.0000056061.18772.72

[41] Levay, E.A., Govic, A., Penman, J., Paolini, A.G. and Kent, S. (2007) Effects of Adult-Onset Calorie Restriction on Anxiety-Like Behavior in Rats. Physiology and Behavior, 92, 889-896.

http://dx.doi.org/10.1016/j.physbeh.2007.06.018 
[42] Geng, Y.Q., Guan, J.T. and Xu, M.Y. (2007) Behavioral Study of Calorie-Restricted Rats from Early Old Age. Proceedings of the 29th Annual International Conference of the IEEE Engineering in Medicine and Biology Society, Lyon, 22-26 August 2007, 2393-2395. http://dx.doi.org/10.1109/iembs.2007.4352809

[43] Geng, Y., Li, T., Liu, X., Li, Z. and Fu, Y. (2011) SIRT1 and SIRT5 Activity Expression and Behavioral Responses to Calorie Restriction. Journal of Cellular Biochemistry, 112, 3755-3761. http://dx.doi.org/10.1002/jcb.23315

[44] Wadden, T.A. and Stunkard, A.J. (1986) Controlled Trial of Very Low Calorie Diet, Behavior Therapy, and Their Combination in the Treatment of Obesity. Journal of Consulting and Clinical Psychology, 54, 482-488. http://dx.doi.org/10.1037/0022-006X.54.4.482

[45] Jahng, J.W., Kim, J.G., Kim, H.J., Kim, B.T., Kang, D.W. and Lee, J.H. (2007) Chronic Food Restriction in Young Rats Results in Depression-and Anxiety-Like Behaviors with Decreased Expression of Serotonin Reuptake Transporter. Brain Research, 1150, 100-107. http://dx.doi.org/10.1016/j.brainres.2007.02.080

[46] Wooley, S.C. and Garner, D.M. (1991) Obesity Treatment: The High Cost of False Hope. Journal of the American Dietetic Association, 91, 1248-1251.

[47] Bellush, L.L., Wright, A.M., Walker, J.P. and Kopchick, J. (1996) Caloric Restriction and Spatial Learning in Old Mice. Physiology and Behavior, 60, 541-547. http://dx.doi.org/10.1016/S0031-9384(96)80029-1

[48] Mamczarz, J., Bowker, J.L., Duffy, K., Zhu, M., Hagepanos, A. and Ingram, D.K. (2005) Enhancement of Amphetamine-Induced Locomotor Response in Rats on Different Regimens of Diet Restriction and 2-Deoxy-D-Glucose Treatment. Neuroscience, 131, 451-464. http://dx.doi.org/10.1016/j.neuroscience.2004.11.019

[49] Rosenzweig, E. and Barnes, C. (2003) Impact of Aging on Hippocampal Function: Plasticity, Network Dynamics, and Cognition. Progress in Neurobiology, 69, 143-179. http://dx.doi.org/10.1016/S0301-0082(02)00126-0

[50] Nehlig, A. and de Vasconcelos, A.P. (1993) Glucose and Ketone Body Utilization by the Brain of Neonatal Rats. Progress in Neurobiology, 40, 163-221. http://dx.doi.org/10.1016/0301-0082(93)90022-K

[51] Zhou, W., Mukherjee, P., Kiebish, M., Markis, W., Mantis, J. and Seyfried, T. (2007) The Calorically Restricted Ketogenic Diet, an Effective Alternative Therapy for Malignant Brain Cancer. Nutrition and Metabolism, 4, 5. http://dx.doi.org/10.1186/1743-7075-4-5

[52] Cao, D., Lu, H., Lewis, T. and Li, L. (2007) Intake of Sucrose-Sweetened Water Induces Insulin Resistance and Exacerbates Memory Deficits and Amyloidosis in a Transgenic Mouse Model of Alzheimer Disease. Journal of Biological Chemistry, 282, 36275-36282. http://dx.doi.org/10.1074/jbc.M703561200

[53] Breier, A., Crane, A.M., Kennedy, C. and Sokoloff, L. (1993) The Effects of Pharmacologic Doses of 2-Deoxy-DGlucose on Local Cerebral Blood Flow in the Awake, Unrestrained Rat. Brain Research, 618, 277-282. http://dx.doi.org/10.1016/0006-8993(93)91276-X 